\title{
GLOBAL EXISTENCE FOR WAVE MAPS WITH TORSION
}

\author{
Stephen C. Anco ${ }^{1}$ and James Isenberg ${ }^{2}$ \\ 1 Department of Mathematics \\ Brock University, St Catharines, ON L2S 3A1, Canada \\ Email: sanco@brocku.ca \\ ${ }^{2}$ Department of Mathematics and Institute of Theoretical Science \\ University of Oregon, Eugene, OR 97403-5203, USA \\ Email: jim@newton.uoregon.edu
}

\begin{abstract}
Wave maps (i.e. nonlinear sigma models) with torsion are considered in $2+1$ dimensions. Global existence of smooth solutions to the Cauchy problem is proven for certain reductions under a translation group action: invariant wave maps into general targets, and equivariant wave maps into Lie group targets. In the case of Lie group targets (i.e. chiral models), a geometrical characterization of invariant and equivariant wave maps is given in terms of a formulation using frames.
\end{abstract}

\section{INTRODUCTION}

There has been considerable progress during the last ten years in the mathematical study of long-time existence properties of solutions of geometricallybased classical field theories. A significant portion of this work has focussed on the study of what are called wave maps in the mathematics literature and nonlinear sigma models (or, in certain special cases, chiral models) in the physics literature. These are defined as maps $\psi$ from a Lorentzian geometry $\left(M^{m+1}, \eta\right)$, e.g. Minkowski space, to a Riemannian geometry $\left(N^{n}, g\right)$, e.g. a symmetric space or a compact Lie group, with $\psi$ being a critical point for 
the functional

$$
S[\psi]=\int_{M^{m+1}} \eta^{\mu \nu} g_{A B}(\psi) \partial_{\mu} \psi^{A} \partial_{\nu} \psi^{B}
$$

and hence satisfying the wave map equation

$$
\eta^{\mu \nu} \nabla_{\mu} \partial_{\nu} \psi^{A}+\Gamma_{B C}^{A}(\psi) \partial_{\mu} \psi^{B} \partial_{\nu} \psi^{C} \eta^{\mu \nu}=0 ;
$$

here $\nabla$ is the (torsion-free) derivative operator determined by the metric $\eta$, and $\Gamma$ are the (torsion-free) connection coefficients compatible with $g$.

Wave maps have a well-posed Cauchy problem, and it is known that for $1+1$ dimensional base geometries $\left(M^{1+1}, \eta\right)$, every choice of smooth initial data evolves into a global smooth solution [1, 2, 3], while for 3+1 (or higher) dimensional base geometries, certain smooth initial data leads to solutions with singularities [3, 国. Not yet understood is what happens in general for $2+1$ dimensional base geometries. This is the "critical dimension" (see [5, 6]), where global smooth solutions are expected, at least, for all smooth initial data of sufficiently small energy. While global existence results are known to hold for certain classes of rotationally-symmetric wave maps in $2+1$ dimensions (without restrictions on the energy) [7, 4], not much is known otherwise for critical wave maps [6].

An interesting modification to the wave map equations can be obtained by adding torsion. This can be done in $2+1$ dimensions, without adding extra dynamical fields, as follows. One fixes a pair of background fields: a closed one-form field $v$ on the base manifold $M^{2+1}$ and a non-closed two-form field $p$ on the target $N^{n}$. The field $p$ serves as a "torsion potential" in the sense that the torsion tensor on $N^{n}$ is defined as

$$
Q_{B C}^{A}=3 / 2 g^{A D} \partial_{[D} p_{B C]} .
$$

A map $\psi: M^{2+1} \rightarrow N^{n}$ is defined to be a torsion wave map if it is a critical point for the functional

$$
S_{\text {tor }}[\psi]=\int_{M^{2+1}}\left(\eta^{\mu \nu} g_{A B}(\psi) \partial_{\mu} \psi^{A} \partial_{\nu} \psi^{B}+\lambda \epsilon^{\mu \nu \sigma} v_{\sigma} p_{A B}(\psi) \partial_{\mu} \psi^{A} \partial_{\nu} \psi^{B}\right)
$$

\footnotetext{
${ }^{1}$ Integrals over $M^{m+1}$ are understood to use the natural volume form compatible with the metric $\eta$.
} 
where $\lambda$ is a coupling constant and $\epsilon$ is the $2+1$ volume tensor normalized with respect to $\eta$. The torsion wave map equation obtained from (4) is given by

$$
\eta^{\mu \nu} \nabla_{\mu} \partial_{\nu} \psi^{A}+\tilde{\Gamma}_{B C}^{A}(\psi) \partial_{\mu} \psi^{B} \partial_{\nu} \psi^{C}\left(\eta^{\mu \nu}+\lambda \epsilon^{\mu \nu \sigma} v_{\sigma}\right)=0
$$

where

$$
\tilde{\Gamma}_{B C}^{A}=\Gamma_{B C}^{A}+Q_{B C}^{A}
$$

are the connection coefficients 1 compatible with $g$, with torsion $Q$. Note that the effect of the torsion is to add the nonlinear term

$$
\lambda \epsilon^{\mu \nu \sigma} v_{\sigma} Q_{B C}^{A}(\psi) \partial_{\mu} \psi^{B} \partial_{\nu} \psi^{C}
$$

to the wave map equation (2).

Wave maps without torsion have a conserved, symmetric stress-energy tensor [5] arising from the functional $S[\psi]$. With the addition of torsion, the corresponding symmetric stress-energy tensor obtained from the functional $S_{\text {tor }}[\psi]$ is no longer conserved. However, we point out that a non-symmetric stress-energy tensor can be derived by considering the variation of $S_{\text {tor }}[\psi]$ under infinitesimal diffeomorphisms of $M^{2+1}$ acting on $\eta, \epsilon, v$, and $\psi$. This leads to

$$
\begin{array}{r}
T_{\alpha}^{\mu}=\eta^{\mu \nu} g_{A B} \partial_{\nu} \psi^{A} \partial_{\alpha} \psi^{B}-1 / 2 \delta^{\mu}{ }_{\alpha} \eta^{\nu \sigma} g_{A B} \partial_{\nu} \psi^{A} \partial_{\sigma} \psi^{B} \\
+1 / 2 \lambda \epsilon^{\mu \nu \sigma} v_{\alpha} p_{A B} \partial_{\nu} \psi^{A} \partial_{\sigma} \psi^{B}
\end{array}
$$

which satisfies

$$
\nabla_{\mu} T_{\alpha}^{\mu}=1 / 2 \lambda \epsilon^{\mu \nu \sigma} \partial_{\mu} \psi^{A} \partial_{\nu} \psi^{B} p_{A B} \nabla_{\alpha} v_{\sigma}
$$

Hence $T^{\mu}{ }_{\alpha}$ is conserved if $v$ is covariantly constant on $\left(M^{2+1}, \eta\right)$. Furthermore, $T^{\mu}{ }_{\alpha}$ reduces to the standard symmetric stress-energy tensor for wave maps without torsion when $v$ is set to zero. The stress-energy tensor (8) is central to investigating global existence for torsion wave maps.

The critical dimension for torsion wave maps, just as for standard wave maps, is $2+1$. While we do not attempt here to investigate the general

\footnotetext{
${ }^{2}$ The contorsion coefficients $\tilde{\Gamma}^{A}{ }_{[B C]}$ compatible with $g$ as determined by the torsion are identically equal to $Q$.
} 
class of critical torsion wave maps, we are able to prove global existence for various reductions of critical wave maps, with and without torsion, where the base geometry is Minkowski space. These reductions are defined by the invariance or equivariance of the wave map $\psi$ under a one-dimensional group of translations acting on $M^{2+1}$. More specifically, choose Cartesian coordinates $(x, y, t)$ for $\left(M^{2+1}, \eta\right)$ and denote the translation group action by $(x, y, t) \rightarrow(x, y+\lambda, t)$. Then, for any target $N^{n}$, a wave map $\psi$ is translation invariant if

$$
\psi^{A}(x, y+\lambda, t)=\psi^{A}(x, y, t) .
$$

Translation equivariant wave maps require that the target $N^{n}$ admit a translation group action. Let $\rho_{B}^{A}(\lambda)$ denote a representation of the translation group action on the base $M^{2+1}$ acting on the target $N^{n}$. Then a wave map $\psi$ is translation equivariant if

$$
\psi^{A}(x, y+\lambda, t)=\rho_{B}^{A}(\lambda) \psi^{B}(x, y, t) .
$$

Note that translation equivariance (11) reduces to translation invariance (10) when (and only when) the representation $\rho(\lambda)$ is chosen to be trivial, $\rho_{B}^{A}(\lambda)=\delta_{B}^{A}$.

One class of targets for which there is a natural translation group action available are Lie groups, $G$. For a Lie group target $N^{n}=G$, left and right multiplication on $G$ by a one-parameter exponential subgroup $\exp (\lambda A)$ define translation group actions, where $A$ is any element in the Lie algebra of $G$. This leads to three types of equivariance as follows. Let $\Psi$ devote a matrix representation of the wave map $\psi: M^{2+1} \rightarrow G$ and let $L$ and $R$ be matrix representations of elements of the Lie algebra of $G$. Then $\psi$ is said to be, respectively, left-translation equivariant if

$$
\Psi(x, y+\lambda, t)=\exp (\lambda L) \Psi(x, y, t),
$$

or right-translation equivariant if

$$
\Psi(x, y+\lambda, t)=\Psi(x, y, t) \exp (\lambda R),
$$

or conjugate-translation equivariant if

$$
\Psi(x, y+\lambda, t)=\exp (\lambda L) \Psi(x, y, t) \exp (\lambda R) .
$$


Corresponding to invariant wave maps (10) and equivariant wave maps (12), (13), (14), we have the following four classes of reductions:

Invariant Wave maps (Any target)

$$
\psi=\phi(x, t)
$$

Left-Equivariant Wave maps (Lie group target)

$$
\Psi=\exp (y L) \Phi_{L}(x, t)
$$

Right-Equivariant Wave maps (Lie group target)

$$
\Psi=\Phi_{R}(x, t) \exp (y R)
$$

Conjugate-Equivariant Wave maps (Lie group target)

$$
\Psi=\exp (y L) \Phi_{C}(x, t) \exp (y R)
$$

In each case the $2+1$ wave map equation for $\psi$ yields a $1+1$ reduced equation for $\phi, \Phi_{L}, \Phi_{R}, \Phi_{C}$, respectively, provided that the target geometry is suitably invariant as discussed later.

We establish global existence of solutions to the Cauchy problem for the class of translation-invariant wave maps with torsion in Section 2. While the proof for these wave maps is very similar to that for $1+1$ wave maps with no torsion, the torsion terms do introduce some subtleties into the analysis, which we highlight.

In order to prove global existence of solutions to the Cauchy problem for the three classes of translation equivariant wave maps with torsion, we find it useful to work with a frame formulation for $2+1$ wave maps . In Section 3 we introduce the frame formulation for general targets and then proceed to relate wave map equivariance for Lie group targets to frame invariance and equivariance. In particular, our global existence theorems for equivariant wave maps have a natural formulation and proof using frames.

The proof for the left equivariant, right equivariant, and conjugate equivariant wave maps with torsion is fairly similar in each case. We focus on the left equivariant case (which corresponds to invariant frames) and carry out the global existence proof in detail, in Section 4. We then briefly note in Section 5 the differences entailed in proving global existence for the other two cases. We make a few concluding remarks in Section 6. 


\section{INVARIANT WAVE MAPS WITH TORSION}

The translation invariance condition (10) is characterized by the wave map functions (15) being independent of $y$. Under this reduction the torsion wave map equation (5) becomes

$$
\gamma^{\alpha \beta} \partial_{\alpha} \partial_{\beta} \phi^{A}+\tilde{\Gamma}_{B C}^{A}(\phi) \partial_{\alpha} \phi^{B} \partial_{\beta} \phi^{C}\left(\gamma^{\alpha \beta}+\lambda v_{y} \epsilon^{\alpha \beta}\right)=0
$$

where $\gamma^{\alpha \beta}$ is the $1+1$ Minkowski metric $(\alpha, \beta$ run over $x$ and $t)$ and $\epsilon^{\alpha \beta}$ is the $1+1$ Levi-Civita tensor. We hereafter take $v_{y}$ to be constant, but we make no further restrictions: The target $\left(N^{n}, g\right)$ can be any Riemannian geometry, and the torsion potential $p$ can be any non-closed two form on $N^{n}$.

Interestingly, while the torsion term

$$
\lambda v_{y} Q_{B C}^{A}(\phi) \partial_{\alpha} \phi^{B} \partial_{\beta} \phi^{C} \epsilon^{\alpha \beta}
$$

appears in a nontrivial way in the reduced wave map equation (19), and while the stress-energy tensor (8) generally contains a torsion term, for translation invariant wave maps the torsion drops out of many of the stress-energy tensor components. We have

$$
\begin{gathered}
T_{t t}=T_{x x}=\frac{1}{2}\left(\left|\partial_{t} \phi\right|^{2}+\left|\partial_{x} \phi\right|^{2}\right), \\
T_{x t}=T_{t x}=\partial_{t} \phi^{A} \partial_{x} \phi^{B} g_{A B},
\end{gathered}
$$

all of which contain no torsion, along with

$$
\begin{gathered}
T_{y y}=\frac{1}{2}\left(\left|\partial_{t} \phi\right|^{2}-\left|\partial_{x} \phi\right|^{2}\right)+\frac{1}{2} \lambda v_{y} \epsilon^{\alpha \beta} \partial_{\alpha} \phi^{A} \partial_{\beta} \phi^{B} p_{A B}, \\
T_{x y}=0 \\
T_{y x}=\frac{1}{2} \lambda v_{x} \epsilon^{\alpha \beta} \partial_{\alpha} \phi^{A} \partial_{\beta} \phi^{B} p_{A B}, \\
T_{t y}=0
\end{gathered}
$$




$$
T_{y t}=\frac{1}{2} \lambda v_{t} \epsilon^{\alpha \beta} \partial_{\alpha} \phi^{A} \partial_{\beta} \phi^{B} p_{A B} .
$$

Note that $v_{x}$ and $v_{t}$ do not appear in the reduced wave map equation (19); setting them to zero does not affect (19), but it does result in $T_{y x}$ and $T_{y t}$ vanishing.

We now consider the Cauchy problem for translation invariant wave maps (15) with torsion. Initial data at $t=t_{0}$ consists of a pair of maps

$$
\hat{\phi}: \Sigma \rightarrow N, \quad \hat{\theta}: \Sigma \rightarrow T N
$$

(here $\Sigma=R^{1}$ or $S^{1}$ allowing for periodic boundary conditions). A solution to the Cauchy problem is then a map $\phi: \Sigma \times R^{1} \simeq M^{2+1} \rightarrow N$ which satisfies (19) along with the initial conditions

$$
\phi\left(x, t_{0}\right)=\hat{\phi}(x), \quad \partial_{t} \phi\left(x, t_{0}\right)=\hat{\theta}(x) .
$$

Note that there are no constraints on the choice of initial data $\{\hat{\phi}, \hat{\theta}\}$. Global existence of initial value solutions is established by the following theorem.

Theorem 1. For any smooth compact support initial data, the Cauchy problem (19) and (29) has a unique smooth global solution $\phi(x, t)$ for all $t \in R^{1}$.

Proof: The PDE system (19) is manifestly hyperbolic; hence, local existence and uniqueness are immediate [9]. To prove global existence, it is sufficient (by the usual open-closed arguments [9]) to show that if $\phi(x, t)$ satisfies (19) on $\Sigma \times I$, with $I$ a bounded open interval in $R^{1}$, then $\phi(x, t)$ and all its derivatives are bounded on $\Sigma \times I$.

To show that $\phi$ and its first derivatives $\partial_{\alpha} \phi$ are bounded, we use an argument based on stress-energy conservation (see [3]). From the form of the stress-energy components (21) to (27), together with the conservation equations

$$
\partial_{t} T^{t}{ }_{t}+\partial_{x} T_{t}^{x}=0, \quad \partial_{t} T^{t}{ }_{x}+\partial_{x} T_{x}^{x}=0,
$$

\footnotetext{
${ }^{3} \hat{\phi}$ is compactly supported if it is constant everywhere outside a compact region in $\Sigma$; $\hat{\theta}$ is compactly supported if it zero outside such a region.
} 
we find that

$$
\gamma^{\alpha \beta} \partial_{\alpha} \partial_{\beta} T_{t t}=0
$$

It then follows from standard results (see [6]) for the wave equation on $1+$ 1 Minkowski space that $T_{t t}$ is bounded on $I$. Thus the first derivatives of $\phi$ are bounded. As a consequence of the mean value theorem and the assumed compact support of the initial data, $\phi$ is then bounded as well.

There are a number of ways of proceeding to argue that second and higher order derivatives of $\phi$ are bounded. Here we use an argument which is adapted from Shatah [6] based on bounding successive $k$ th order energies

$$
\mathcal{E}_{k}(t)=\frac{1}{2} \int_{\Sigma}\left(\left|\partial_{t} \partial_{x}{ }^{k} \phi\right|^{2}+\left|\partial_{x}{ }^{k+1} \phi\right|^{2}\right) d x
$$

Note that the ordinary energy

$$
\mathcal{E}_{0}(t)=\int_{\Sigma} T_{t t} d x=\frac{1}{2} \int_{\Sigma}\left(\left|\partial_{t} \phi\right|^{2}+\left|\partial_{x} \phi\right|^{2}\right) d x
$$

is bounded and independent of $t, \mathcal{E}_{0}(t)=\mathcal{E}_{0}\left(t_{0}\right)$, for smooth compact support initial data.

We start by rewriting the torsion wave map equation (19) in the form

$$
D^{\alpha} V_{\alpha}^{A}=0
$$

where $V_{\alpha}^{A}=\partial_{\alpha} \phi^{A}, D^{\alpha}=\gamma^{\alpha \beta} D_{\beta}$, and $D_{\beta}=\partial_{\beta}+\Gamma^{A}{ }_{B C} V_{\beta}^{C}+\lambda Q^{A}{ }_{B C} \epsilon_{\beta}{ }^{\alpha} V_{\alpha}^{C}$ defines a covariant derivative operator which includes the connection with torsion. If we now apply $D_{\beta}$ to equation (34) and commute $D_{\beta}$ past the derivative operators, keeping track of the various curvature and torsion terms which arise, then we obtain a nonlinear wave equation for $V_{\alpha}^{A}$ :

$$
D^{\alpha} D_{\alpha} V_{\beta}^{A}+P_{\beta}^{A}(V, V, V)=0
$$

where $P(V, V, V)$ denotes an expression which is trilinear in $V_{\beta}{ }^{A}$ and involves no higher derivatives of $\phi^{A}$.

By multiplying (35) by $\gamma^{\alpha \beta} g_{C A} D_{t} V_{\alpha}^{C}$, we straightforwardly derive the conservation equation

$$
D_{t}\left(\frac{1}{2}\left|D_{t} V\right|^{2}+\frac{1}{2}\left|D_{x} V\right|^{2}\right)-D_{x}\left(D_{t} V \cdot D_{x} V\right)=\tilde{P}(V, V, V) \cdot D V
$$


where $\tilde{P}(V, V, V)$ is, like $P(V, V, V)$, trilinear in $V$ with no higher derivatives of $\phi^{A}$. Now, integrating (36) over $\Sigma$, we obtain

$$
\partial_{t} \mathcal{E}_{1}(t)=\int_{\Sigma} \tilde{P}(V, V, V) \cdot D V d x
$$

for the 1st order energy defined in (32). Estimating the right hand side of (37), we find

$$
\begin{aligned}
\partial_{t} \mathcal{E}_{1}(t) \leq & C\|V\|_{L^{6}}^{3}\left\|D_{\alpha} V\right\|_{L^{2}} \\
& \leq C\|V\|_{L^{6}}^{3} \sqrt{\mathcal{E}_{1}(t)}
\end{aligned}
$$

and hence

$$
\partial_{t} \sqrt{\mathcal{E}_{1}} \leq C\|V\|_{L^{6}}^{3}
$$

It follows from Sobolev inequalities that

$$
\|V\|_{L^{6}} \leq C\|V\|_{L^{2}}^{2 / 3}\|D V\|_{L^{2}}^{1 / 3}
$$

so we have

$$
\partial_{t} \sqrt{\mathcal{E}_{1}} \leq C\|V\|_{L^{2}}^{2}\|D V\|_{L^{2}} \leq C \mathcal{E}_{0} \sqrt{\mathcal{E}_{1}}
$$

Since $\mathcal{E}_{0}$ is bounded, it follows from (41) that

$$
\sqrt{\mathcal{E}_{1}(t)} \leq C e^{k t}
$$

which bounds $\mathcal{E}_{1}(t)$, and therefore bounds the $L^{2}$ norm of $D V$. Hence $\left\|\partial_{\alpha}^{2} \phi\right\|_{L^{2}}$ is bounded.

To bound $\mathcal{E}_{2}(t)$, we start from the wave equation (35) for $V$ and repeat the previous argument. Setting $W_{\beta \gamma}{ }^{A}:=D_{\beta} V_{\gamma}{ }^{A}$, we derive

$$
D^{\alpha} D_{\alpha} W_{\beta \gamma}{ }^{A}+R_{\beta \gamma}{ }^{A}(V, V, W)=0
$$

where $R(V, V, W)$ is bilinear in $V$, linear in $W$, and involves no other derivatives of $\phi$. From (43) we obtain the conservation equation

$$
D_{t}\left(\frac{1}{2}\left|D_{t} W\right|^{2}+\frac{1}{2}\left|D_{x} W\right|^{2}\right)-D_{x}\left(D_{t} W \cdot D_{x} W\right)=\tilde{R}(V, V, W) \cdot D W
$$


where $\tilde{R}(V, V, W)$ has the same properties as $R(V, V, W)$. Integrating over $\Sigma$ and estimating, we obtain

$$
\partial_{t} \mathcal{E}_{2}(t) \leq C\|V\|_{L^{8}}^{2}\|W\|_{L^{4}} \sqrt{\mathcal{E}_{2}(t)}
$$

Since $V$ and $W=D V$ are $L^{2}$ bounded, by applying Sobolev inequalities to (45) we obtain

$$
\partial_{t} \sqrt{\mathcal{E}_{2}} \leq C \sqrt{\mathcal{E}_{2}}
$$

Hence we have that $\mathcal{E}_{2}(t)$ is bounded and therefore so is the $L^{2}$ norm of $D W$. Thus, since $D W=D D V$, it follows that $\left\|\partial_{\alpha}^{3} \phi\right\|_{L^{2}}$ is bounded.

The argument proceeds to all successively higher orders and we thereby determine that all derivatives of $\phi$ are $L^{2}$ bounded. It follows from Sobolev embedding that all derivatives of $\phi$ are pointwise bounded, which completes the proof of Theorem 1.

\section{EQUIVARIANT WAVE MAPS WITH TORSION AND THE FRAME FORMULATION}

We begin by setting up a frame formulation for wave maps with and without torsion. (See also [7]). We first choose a frame basis $\left\{e_{a}^{A}\right\}(a=1, \ldots, n)$ for the target geometry $\left(N^{n}, g\right)$ and let $e_{A}^{a}(\psi)$ denote the frame associated to $\psi$. We now define the "frame fields"

$$
K_{\mu}^{a}:=e_{A}^{a}(\psi) \partial_{\mu} \psi^{A}
$$

where $\left\{e_{A}^{a}\right\}$ are the components of the dual basis to $\left\{e_{a}^{A}\right\}$. These frame fields $K_{\mu}^{a}$ may be viewed either as the pull-back of the dual frame $\left\{e_{A}^{a}\right\}$ from $N^{n}$ to $M^{2+1}$ along the map $\psi: M^{2+1} \rightarrow N^{n}$, or as the frame components of the wave map gradient on the tangent space of the target geometry $\left(N^{n}, g\right)$. In any case, it follows from (47) that $K$ satisfies the identity

$$
\nabla_{[\nu} K_{\mu]}^{a}=-1 / 2 C_{b c}{ }^{a}(\psi) K_{\nu}^{b} K_{\mu}^{c}
$$

where $C_{b c}{ }^{a}$ are the frame commutator coefficients defined by

$$
\left[e_{b}, e_{c}\right]=C_{b c}{ }^{a} e_{a}
$$


Moreover, one verifies that if $\psi$ satisfies the wave map equation (21), then $K$ satisfies

$$
\nabla^{\mu} K_{\mu}^{a}=-C_{b c}^{a}(\psi) K_{\nu}^{b} K_{\mu}^{c} \eta^{\nu \mu}
$$

where $C^{a}{ }_{b c}:=g^{a d} C_{d b}{ }^{e} g_{e c}$, with $g^{a d}:=e_{A}^{a} e_{B}^{b} g^{A B}$ and $g_{a b}:=e_{a}^{A} e_{b}^{B} g_{A B}$; or if $\psi$ satisfies the torsion wave map equation (5), then $K$ satisfies

$$
\nabla^{\mu} K_{\mu}^{a}=-C_{b c}^{a}(\psi) K_{\nu}^{b} K_{\mu}^{c} \eta^{\nu \mu}-\lambda \epsilon^{\sigma \nu \mu} v_{\sigma} Q_{b c}^{a}(\psi) K_{\nu}^{b} K_{\mu}^{c}
$$

where $Q_{b c}^{a}:=e_{A}^{a} Q^{A}{ }_{B C} e_{b}^{B} e_{c}^{C}$.

Up to this point in setting up the frame formulation, we have made no restrictions on the choice of the target or on the nature of the wave maps. We now focus on equivariant wave maps (12) to (14) and their corresponding frame formulations, so we assume the target geometry to be a Lie group $G$. While $K$ can be defined for any frame basis on $G$, the frame field equations are simplest if we require that $\left\{e_{a}^{A}\right\}$ be a left-invariant basis for $G$. It then follows that the commutator coefficients $C_{b c}{ }^{a}$ are independent of $\psi$ and are constant. If we make the further restrictions that the metric $g$ be a leftinvariant tensor on $G$,

$$
g_{A B}=e^{a}{ }_{A} e^{b}{ }_{B} g_{a b},
$$

and the torsion potential $p$ be a left invariant two-form on $G$,

$$
p_{A B}=e^{a}{ }_{A} e^{b}{ }_{B} p_{a b},
$$

so that the components $g_{a b}$ and $p_{a b}$ are constant, then the coefficients $C_{b c}^{a}(\psi)$ are independent of $\psi$ and constant while so are the frame components $Q^{a}{ }_{b c}(\psi)$ as well; in particular, we have

$$
Q_{b c}^{a}=-3 / 2 g^{a d} p_{e[d} C_{b c]}^{e}
$$

with

$$
C_{b c}{ }^{a}=2 e_{A}^{a} e_{[b \mid}^{B} \partial_{B} e_{\mid c]}^{A} .
$$

Remark 1: Every nonabelian Lie group admits both a left-invariant metric $g$ and a left-invariant two-form $p$. However, for semi-simple Lie groups $G$, if $G$ has dimension three then all left-invariant two-forms $p$ are necessarily 
closed, and consequently $Q=0$ so there is no torsion. This is not the case if $G$ has larger dimension. In particular, a non-closed left-invariant two-form $p$ and hence non-zero torsion $Q$ is admitted by all nonabelian semi-simple Lie groups $G$ other than the three-dimensional ones (namely $S U(2)$ and its real forms $S O(3), S O(1,2), S O(2,1))$. See Proposition A in the appendix.

We now find that, assuming the restrictions just noted, we can write equations (48), (50) and (51) strictly in terms of the frame fields $K$, with no explicit $\psi$ dependence:

$$
\begin{aligned}
& \nabla_{[\nu} K^{a}{ }_{\mu]}=-1 / 2 C_{b c}{ }^{a} K_{\nu}{ }^{b} K_{\mu}^{c}, \\
& \nabla^{\mu} K_{\mu}^{a}=-C^{a}{ }_{b c} K^{b}{ }_{\nu} K_{\mu}^{c} \eta^{\nu \mu} \\
& \nabla^{\mu} K_{\mu}{ }^{a}=-C^{a}{ }_{b c} K^{b}{ }_{\nu} K_{\mu}^{c} \eta^{\nu \mu}-\lambda \epsilon^{\sigma \nu \mu} v_{\sigma} Q^{a}{ }_{b c} K^{b}{ }_{\nu} K_{\mu}^{c} .
\end{aligned}
$$

The field equations (56) and(57) together are a self-contained PDE system for $K$ which is equivalent to the wave map equation (2); the field equations (56) and(58) likewise are a self-contained PDE system for $K$ which is equivalent to the wave map equation with torsion (5). Note that the system with torsion reduces to the system without torsion when $\lambda=0$.

Proposition 1. Let $\left(M^{2+1}, \eta\right)$ be a Lorentzian geometry, and let $N^{n}=G$ be a Lie group target.

1. Suppose that $\psi^{A}$ is a solution of the torsion wave map equation (5). Then $K_{\mu}^{a}$ defined by (47) satisfies the field equations (50) and (58).

2. Suppose that $K_{\mu}^{a}$ is a solution of the field equations (50) and (58). If $M^{2+1}$ is simply connected, then there exists a torsion wave map $\psi^{A}$, satisfying equation (5), which is related to $K_{\mu}^{a}$ by (䅦).

Proof: To prove part (1), we first note that for $K^{a}{ }_{\mu}$ given by (47), the field equation (56) is an identity. We then verify that, through the torsion wave map equation (5), the substitution of (47) for $K_{\mu}^{a}$ satisfies the field equation (58).

For the converse, to prove part (2), we note the field equation (56) shows that $K_{\mu}^{a}$ can be viewed as a Lie-algebra valued connection one-form on the trivial bundle $M^{2+1} \times G$, with zero curvature. Since the bundle is trivial and the manifold $M^{2+1}$ is assumed to be simply connected, there exists a global 
parallel section. Correspondingly, there exists a smooth map $U: M^{2+1} \rightarrow G$ (called a "gauge transformation") in terms of which we have

$$
K_{\mu}^{a}=\left(U^{-1} \partial_{\mu} U\right)^{A} e_{A}^{a}(I)
$$

where $I$ is the identity element of $G$.

Now let $\psi^{A}$ denote $U$ written in terms of local coordinates on $G$. It follows that $U^{-1}$ pulls back $e_{A}^{a}(I)$ to $e_{A}^{a}(\psi)$, at the Lie group element specified by $U$. Hence we have

$$
\left(U^{-1} \partial_{\mu} U\right)^{A} e_{A}^{a}(I)=e_{A}^{a}(\psi) \partial_{\mu} \psi^{A}
$$

Combining (60) with (59), we obtain equation (47). Then by substituting (47) into the field equation (58), we verify that $\psi$ satisfies (5).

We note that, independent of their usefulness for the study of wave maps, these field theories in terms of $K$ viewed as a Lie-algebra valued one-form field on $M^{2+1}$ have some interest as a nonlinear generalization of Maxwell's equations. Indeed, for the abelian case $C_{a b}{ }^{c}=0$, the field equations (56) and (57) are exactly Maxwell's equations in $2+1$ dimensions, while the field equations (56) and (58) are a modification of Maxwell's equations by adding torsion. This relationship is explored elsewhere [10, 11, 12].

Since we will use frame fields to study translation equivariant wave maps, we now characterize frame fields which correspond to the three classes of equivariant wave maps (12), (13), (14). We begin with the following definitions of invariant and equivariant frame fields under a translation group action.

\section{Invariant Frame Field:}

$$
K(x, y+\lambda, t)=K(x, y, t)
$$

\section{Equivariant Frame Field:}

$$
K(x, y+\lambda, t)=\exp (-\lambda A) K(x, y, t) \exp (\lambda A)
$$

Here $A$ is an element of the Lie algebra of the target Lie group $G$, and $(x, y, t)$ are standard coordinates for the Minkowski space base geometry $\left(M^{2+1}, \eta\right)$.

Geometrically, the translation equivariant group action (62) on $K$ arises via the pull-back of the dual frame components $\left\{e_{A}^{a}\right\}$ under right multiplication in $G$ by the one-parameter exponential subgroup generated from the Lie 
algebra element $R$. When $R=0$ this group action reduces to the translation invariant group action (61) on $K$. (Alternatively, note that the translation invariant group action arises directly by left multiplication in $G$ since the dual frame is left-invariant.)

Based on Proposition 1, the correspondence between invariant/equivariant frame fields and wave maps is summarized by the following two results.

\section{Proposition 2.}

1. If $\psi$ is left equivariant (12), then the corresponding frame field $K$ is invariant (61).

2. If $\psi$ is right equivariant (19), then the corresponding frame field $K$ is equivariant (62), with the components $K_{y}^{a}$ constant.

3. If $\psi$ is conjugate equivariant (14), then the corresponding frame field $K$ is equivariant (62).

The proof of these correspondences amounts to a direct calculation using a matrix representation for $\psi$ and $K$. There are straightforward converse correspondences as well.

\section{Proposition 3.}

1. If $K$ is invariant (61), then the corresponding wave map $\psi$ is left equivariant $(19)$.

2. If $K$ is equivariant (69), then the corresponding wave map $\psi$ is conjugate equivariant (14).

3. If $K$ is equivariant (69) with the components $K_{y}^{a}$ constant, then the corresponding wave map $\psi$ is right equivariant (13).

Proof: Let $U$ denote a the matrix representation of the wave map $\psi$ corresponding to $K$. We first prove part (1). It follows from the definition of frame field invariance, together with relation (59), that $U$ satisfies

$$
\partial_{y}\left(U^{-1} \partial_{\mu} U\right)=0
$$


Integrating the $y$ component of this equation, and then multiplying both sides by $U$, we obtain the linear matrix ordinary differential equation

$$
\partial_{y} U(x, y, t)=U(x, y, t) f(x, t)
$$

where $f$ is an arbitrary Lie-algebra matrix valued function (independent of $y)$. The general solution to (64) is

$$
U(x, y, t)=\exp (y A(x, t)) V(x, t)
$$

where $V$ is an arbitrary Lie-algebra nonsingular matrix valued function (independent of $y$ ), and $A:=V f V^{-1}$.

We now impose the $x, t$ components of equation (63). Calculating $U^{-1} \partial_{t} U$ with $U$ from (65), we find

$$
U^{-1} \partial_{t} U=V^{-1} \partial_{t} V+V^{-1} \exp (-y A)\left(\partial_{t} \exp (y A)\right) V
$$

SO

$$
0=\partial_{y}\left(U^{-1} \partial_{t} U\right)=V^{-1} \exp (-y A) \partial_{t} A \exp (y A) V
$$

which implies that

$$
\partial_{t} A=0
$$

Similarly, working with $U^{-1} \partial_{x} U$ and imposing $0=\partial_{y}\left(U^{-1} \partial_{x} U\right)$ we determine that

$$
\partial_{x} A=0 .
$$

Thus $A$ must be a constant Lie-algebra valued matrix, which we denote $L$; then (65) becomes

$$
U(x, y, t)=\exp (y L) V(x, t) .
$$

Condition (12) immediately follows, so the wave map corresponding to $K$ is left equivariant.

We now prove part (2). From the definition of frame equivariance, there is a $y$-independent Lie-algebra matrix valued field $f_{\mu}(x, t)$ and a constant Lie-algebra matrix $A$ such that

$$
K_{\mu}(x, y, t)=\exp (-y A) f_{\mu}(x, t) \exp (y A) .
$$


Hence, from relation (59), $U(x, y, t)$ must satisfy

$$
U^{-1} \partial_{\mu} U=\exp (-y A) f_{\mu} \exp (y A) .
$$

The $y$-component of this equation yields

$$
\left(\partial_{y} U\right) \exp (-y A)=U \exp (-y A) f_{y},
$$

and after some manipulation we obtain the linear matrix ODE

$$
\partial_{y} W=W\left(f_{y}-A\right)
$$

where $W(x, y, t):=U(x, y, t) \exp (-y A)$. The general solution to (74) is

$$
W(x, y, t)=\exp (y B(x, t)) V(x, t)
$$

where $V$ is an arbitrary Lie-algebra matrix valued function, and $B$ is defined as

$$
B:=V\left(f_{y}-A\right) V^{-1}
$$

Working with the other components of equation $(72)$ we derive

$$
\partial_{t} W=W f_{t}
$$

and

$$
\partial_{x} W=W f_{x} .
$$

Then rearranging (77) and using (75), we obtain

$$
f_{t}(x, t)=W^{-1} \partial_{t} W=V^{-1} \partial_{t} V+V^{-1} \exp (-y B) \partial_{t}(\exp (-y B)) V .
$$

Since both $f_{t}$ and $V$ are independent of $y$, if we take $\partial_{y}$ of both sides of equation (79) we have

$$
\begin{aligned}
0 & =\partial_{y}\left(V^{-1} \exp (-y B) \partial_{t}(\exp (y B)) V\right) \\
& =V^{-1} \exp (-y B) \partial_{t} B \exp (y B) V
\end{aligned}
$$

which implies that $\partial_{t} B=0$. Similarly, using (78), we find that $\partial_{x} B=0$. Hence $B$ is a constant Lie-algebra matrix, which we denote $L$. Thus, after combining (75) with the definition $W=U \exp (-y A)$, we see that

$$
U(x, y, t)=\exp (y L) V(x, t) \exp (y A)
$$


so $U(x, y, t)$ is conjugate equivariant (14) with $R=A$.

Finally, we prove part (3). From (81) we have

$$
K_{y}=U^{-1} \partial_{y} U=\exp (-y A) V^{-1} L V \exp (y A)+A
$$

which is assumed to be constant. By differentiating with respect to $y$, we obtain $\left[V^{-1} L V, A\right]=0$, and hence (81) becomes $K_{y}=V^{-1} L V+A$. Thus, it follows that $B:=V^{-1} L V$ defines a constant Lie-algebra matrix which commutes with $A$. We then have

$$
\begin{aligned}
U(x, y, t) & =\exp (y L) V(x, t) \exp (y A) \\
& =V(x, t) \exp (y B) \exp (y A)=V(x, t) \exp (y R)
\end{aligned}
$$

where $R:=A+B$. Hence $U(x, y, t)$ is right equivariant (13).

As a consequence of Propositions 2 and 3, we can prove global existence of solutions to the Cauchy problem for the three classes of translation equivariant wave maps (with or without torsion) by using invariant or equivariant frame fields. We do this first for the invariant frame fields in the next section.

Our analysis makes essential use of the wave map stress-energy tensor (8). Through the relation (47) for $K$ in terms of $\psi$, we obtain

$$
T^{\mu}{ }_{\alpha}=\eta^{\mu \nu} K_{\nu}^{a} K_{\alpha}^{b} g_{a b}-1 / 2 \delta^{\mu}{ }_{\alpha} \eta^{\nu \sigma} K_{\nu}^{a} K_{\sigma}^{b} g_{a b}+1 / 2 \lambda \epsilon^{\mu \nu \sigma} v_{\alpha} p_{a b} K_{\nu}^{a} K_{\sigma}^{b} .
$$

One verifies that, for solutions $K$ of (56) and (58) in which $\left(M^{2+1}, \eta\right)$ is Minkowski space, this non-symmetric stress-energy tensor satisfies the conservation equation

$$
\partial_{\mu} T_{\alpha}^{\mu}=1 / 2 \lambda \epsilon^{\mu \nu \sigma} p_{a b} K_{\mu}^{a} K_{\nu}^{b} \partial_{\alpha} v_{\sigma} .
$$

Hereafter we specialize to the situation where $v$ is constant on $M^{2+1}$. This makes the analysis of the field equations considerably simpler. In particular, the stress-energy is strictly conserved, $\partial_{\mu} T^{\mu}{ }_{\alpha}=0$.

\section{GLOBAL EXISTENCE FOR INVARIANT FRAME FIELD EQUATIONS WITH TORSION}

By definition (61) of translation invariance for frame fields, the component functions $K_{\mu}^{a}$ are independent of $y$. Then, adopting the convenient notation

$$
E^{a}:=K_{x}^{a}, H^{a}:=K_{y}^{a}, B^{a}:=K_{t}^{a},
$$


we find that the translation-invariant frame field equations take the form

$$
\begin{aligned}
\partial_{x} H^{a}= & -C_{b c}{ }^{a} E^{b} H^{c} \\
\partial_{t} E^{a}= & \partial_{x} B-C_{b c}{ }^{a} B^{b} E^{c} \\
\partial_{t} H^{a}= & -C_{b c}{ }^{a} B^{b} H^{c} \\
\partial_{t} B^{a}= & \partial_{x} E^{a}-C^{a}{ }_{b c}\left(B^{b} B^{c}-E^{b} E^{c}-H^{b} H^{c}\right) \\
& \quad-\lambda Q^{a}{ }_{b c}\left(v_{y} B^{b} E^{c}-v_{x} B^{b} H^{c}+v_{t} E^{b} H^{c}\right)
\end{aligned}
$$

for the functions $\left\{E^{a}(x, t), H^{a}(x, t), B^{a}(x, t)\right\}$. Note that, in this system of field equations, (87) is a constraint equation while (88) to (90) are evolution equations.

Initial data at $t=t_{0}$ for the Cauchy problem is specified by choosing (on $\Sigma=R^{1}$ or $S^{1}$ allowing for periodic boundary conditions) Lie-algebra valued functions $\left\{\hat{E}^{a}(x), \hat{H}^{a}(x), \hat{B}^{a}(x)\right\}$ which satisfy the constraint

$$
\partial_{x} \hat{H}^{a}=-C_{b c}{ }^{a} \hat{E}^{b} \hat{H}^{c} .
$$

A solution to the Cauchy problem is then a set of fields $\left\{E^{a}(x, t), H^{a}(x, t)\right.$, $\left.B^{a}(x, t)\right\}$ satisfying (88) to (90) and the initial conditions

$$
E^{a}\left(x, t_{0}\right)=\hat{E}^{a}(x), H^{a}\left(x, t_{0}\right)=\hat{H}^{a}(x), B^{a}\left(x, t_{0}\right)=\hat{B}^{a}(x) .
$$

To show that the Cauchy problem is well-posed, we note that well-posedness is known for the wave map equation without torsion [6], which is equivalent to the system (87) to (90) up to the addition of the torsion terms involving $\lambda$. These terms do not involve any derivatives of the fields and hence do not effect the well-posedness. Alternatively, we note that, up to such terms, the system is equivalent to the Maxwell equations in $2+1$ dimensions, which constitute a well-posed system. It follows that the system (87) to (90) is well-posed and, moreover, is first-order hyperbolic.

In this section we prove global existence of smooth solutions to the Cauchy problem for the $1+1$ field equations (87) to (90). The proof relies on the use of the stress-energy tensor (84) along with light cone estimates.

To proceed we write out the components of the stress-energy tensor (8) in terms of $E^{a}, H^{a}, B^{a}$. Using the coordinates $(x, y, t)$ for $M^{2+1}$ we have

$$
T_{x x}=\frac{1}{2}\left(E_{x}^{2}-E_{y}^{2}+B^{2}\right)+\lambda v_{x} H^{a} B^{b} p_{a b}
$$




$$
\begin{aligned}
& T_{y y}=\frac{1}{2}\left(-E_{x}^{2}+E_{y}^{2}+B^{2}\right)+\lambda v_{y} B^{a} E^{b} p_{a b} \\
& T_{t x}=E \cdot B+\lambda v_{x} H^{a} E^{b} p_{a b} \\
& T_{x t}=E \cdot B+\lambda v_{t} H^{a} B^{b} p_{a b} \\
& T_{t y}=H \cdot B+\lambda v_{y} H^{a} E^{b} p_{a b} \\
& T_{y t}=H \cdot B+\lambda v_{t} B^{a} E^{b} p_{a b} \\
& T_{x y}=E \cdot H+\lambda v_{y} H^{a} B^{b} p_{a b} \\
& T_{y x}=E \cdot H+\lambda v_{x} B^{a} E^{b} p_{a b}
\end{aligned}
$$

where $E^{2}:=E^{a} E^{b} g_{a b}$ and $E \cdot B=E^{a} B^{b} g_{a b}$, etc..

For derivation of light cone estimates, it is useful to work with null components of the stress-energy tensor. We introduce null coordinates which mix $t$ and $x$ (but not $y)$ :

$$
\begin{gathered}
\ell=t+x \\
n=-t+x
\end{gathered} \longleftrightarrow \begin{aligned}
& x=\frac{1}{2}(\ell+n) \\
& t=\frac{1}{2}(\ell-n)
\end{aligned}
$$

Then we find (for the components we will need):

$$
\begin{aligned}
T_{\ell \ell} & =K_{\ell}^{2}+\lambda v_{\ell} H^{a} K_{\ell}^{b} p_{a b} \\
& =\frac{1}{4}(B+E)^{2}+\frac{\lambda}{4}\left(v_{t}+v_{x}\right) H^{a}\left(B^{b}+E^{b}\right) p_{a b} \\
T_{n n}= & K_{n}^{2}-\lambda v_{n} H^{a} K_{n}^{b} p_{a b} \\
= & \frac{1}{4}(-B+E)^{2}-\frac{\lambda}{4}\left(-v_{t}+v_{x}\right) H^{a}\left(-B^{b}+E^{b}\right) p_{a b} \\
T_{\ell n} & =-\frac{1}{2} K_{y}^{2}-\lambda v_{n} K_{\ell}^{a} H^{b} p_{a b} \\
& =-\frac{1}{2} E_{y}^{2}+\frac{\lambda}{4}\left(-v_{t}+v_{x}\right) H^{a}\left(B^{b}+E^{b}\right) p_{a b} \\
T_{n \ell} & =-\frac{1}{2} K_{y}^{2}+\lambda v_{\ell} K_{n}^{a} H^{b} p_{a b} \\
& =-\frac{1}{2} E_{y}^{2}-\frac{\lambda}{4}\left(v_{t}+v_{x}\right) H^{a}\left(-B^{b}+E^{b}\right) p_{a b} .
\end{aligned}
$$


For these components the stress-energy conservation equation (85) has the null component form

$$
\begin{aligned}
& \partial_{n} T_{\ell \ell}+\partial_{\ell} T_{n \ell}=0 \\
& \partial_{\ell} T_{n n}+\partial_{n} T_{\ell n}=0 .
\end{aligned}
$$

These equations are essential for the derivation of the light cone estimates we will need.

Also important for our analysis is the energy function

$$
\begin{aligned}
\mathcal{E}(t) & =\int_{\Sigma} T_{t t} d x \\
& =\int_{\Sigma}\left(\frac{1}{2}\left(E^{2}+H^{2}+B^{2}\right)+\lambda v_{t} H^{a} E^{b} p_{a b}\right) d x
\end{aligned}
$$

We note that for certain values of the coupling constant $\lambda$, the energy $\mathcal{E}(t)$ can be negative, and it therefore does not in general control the $L^{2}$ norm of $E^{a}, H^{a}$, or $B^{a}$. However, for sufficiently small $\lambda$, there is a constant $k>0$ such that

$$
\frac{1}{k}\left(E_{x}^{2}+E_{y}^{2}\right) \leq E_{x}^{2}+E_{y}^{2}+2 \lambda v_{t} H^{a} E^{b} p_{a b} \leq k\left(E_{x}^{2}+E_{y}^{2}\right)
$$

and hence the energy is positive, so that $\mathcal{E}(t)$ does consequently control $\|E\|_{L^{2}},\|H\|_{L^{2}}$, and $\|B\|_{L^{2}}$. We assume henceforth that $\lambda$ is sufficiently small for this to be the case. T

We now state our main results. Let $\Sigma$ denote $R^{1}$ or $S^{1}$, and introduce coordinates $(x, t)$ for $\Sigma \times R^{1} \simeq M^{2+1}$. Fix constants $v_{t}, v_{x}, v_{y}$. Let $G$ be a Lie group with $C_{b c}{ }^{a}$ denoting the Lie-algebra commutator structure tensor. Fix on the Lie algebra of $G$ a positive definite metric tensor $g_{a b}$ (it need not necessarily be compatible with the commutator) and a skew-tensor $p_{a b}$. Let $Q_{b c}^{a}$ be the tensor defined by (54).

\footnotetext{
${ }^{4}$ It is sufficient that $\lambda$ satisfy
}

$$
|\lambda| \leq 1 / \sqrt{\left|v_{t}\right||p|}
$$

where $|p|^{2}=\left|p_{a b} p_{c d} g^{a c} g^{b d}\right|$. 
Theorem 2. Let $\lambda$ be a small constant. ${ }^{4}$ For any smooth compact support initial data (99) satisfying (91), the Cauchy problem (87) to (90) has a unique smooth global solution $\left\{E^{a}(x, t), H^{a}(x, t), B^{a}(x, t)\right\}$ for all $t \in R^{1}$

Combining this result with Propositions 2 and 3 from Section 3, we have a corresponding result for wave maps.

Theorem 3. The Cauchy problem for left-translation equivariant Lie group wave maps (19), with or without torsion, has a unique smooth global solution for all smooth compact support initial data.

\section{Proof of Theorem 2:}

Local existence and uniqueness of smooth solutions of the PDE system (87) to (99) follows from standard results (see, for example, [9]) for first-order hyperbolic systems in $1+1$ dimensions. In order to prove global existence, it is sufficient by the usual "open-closed" arguments [9] to establish the following: For $\left\{E^{a}(x, t), H^{a}(x, t), B^{a}(x, t)\right\}$ satisfying equations (87) to (90) for $t \in I$, with $I$ a bounded open interval in $R^{1}$, each component of these fields is bounded for $t \in I$, as are all orders of their derivatives. We prove this boundedness result as follows:

\section{Step 1: Conserved Energy}

It follows from the stress-energy conservation equation $\partial_{t} T_{t t}-\partial_{x} T_{x t}=0$ that the energy $\mathcal{E}(t)$ satisfies $\frac{d}{d t} \mathcal{E}(t)=\mathcal{F}(t)$ where

$$
\mathcal{F}(t):=\int_{\Sigma} \partial_{x} T_{x t} d x=\left.T_{x t}\right|_{\partial \Sigma}
$$

is the flux. If we are working on $\Sigma=S^{1}$, then $\partial \Sigma$ is empty, so $\mathcal{F}(t)=0$. If instead $\Sigma=R^{1}$, then we note that as a consequence of hyperbolicity of the system (87) to (90), the fields $\left\{E^{a}, H^{a}, B^{a}\right\}$ have compact support on $\Sigma$ for all $t \in I$, and hence $\mathcal{F}(t)=0$. Thus, the energy is conserved, $\mathcal{E}(t)=\mathcal{E}\left(t_{0}\right)$, for all $t \in I$.

As we noted earlier, the energy controls the $L^{2}$ norm of the fields $\left\{E^{a}\right.$, $\left.H^{a}, B^{a}\right\}$, so long as $\lambda$ is sufficiently small (as assumed in the theorem). Hence we have

$$
\left\|E^{a}\right\|_{L^{2}(\Sigma)}<k, \quad\left\|H^{a}\right\|_{L^{2}(\Sigma)}<k, \quad\left\|B^{a}\right\|_{L^{2}(\Sigma)}<k
$$


for some constant $k$ (depending on $\mathcal{E}\left(t_{0}\right)$ ), for all $t \in I$.

\section{Step 2: Bounded $H^{a}$}

In the system (87) to (90), the field $H^{a}$ enters in a different way from $E^{a}$ and $B^{a}$, since the evolution equation (89) for $H^{a}$ involves no spatial derivative terms, and the constraint equation (87) has $\partial_{x} H^{a}$ appearing, but no spatial derivatives of $E^{a}$ or $B^{a}$. Consequently, we treat $H^{a}$ differently from the other two fields: we first show that $H^{a}$ is bounded, and then use this in showing that $E^{a}$ and $B^{a}$ are bounded.

To start, we integrate the absolute values of both sides of the constraint equation (87) over $\Sigma$, obtaining

$$
\int_{\Sigma}\left|\partial_{x} H^{a}\right| d x=\int_{\Sigma}\left|C_{b c}^{a} E^{b} H^{c}\right| d x .
$$

Since $C_{b c}{ }^{a}$ is constant, there exists a constant $k_{1}$ such that

$$
\left|C_{b c}{ }^{a} E^{b} H^{c}\right| \leq\left|C_{b c}{ }^{a}\right|\left|E^{b}\right|\left|H^{c}\right| \leq k_{1}\left(E^{2}+H^{2}\right)
$$

by standard algebraic inequalities. It follows from (112) and (113) together with the bounds (111) that

$$
\int_{\Sigma}\left|\partial_{x} H^{a}\right| d x \leq k_{2}
$$

for a constant $k_{2}$. Combining (114) with the mean value theorem, we obtain controls on the spatial variation of $E_{y}(x, t)$ for any fixed time $t$. In particular, for any $x_{1}, x_{2} \in \Sigma$ with fixed $t$, we have

$$
\begin{aligned}
\left|H^{a}\left(x_{2}, t\right)-H^{a}\left(x_{1}, t\right)\right| & =\left|\int_{x_{1}}^{x_{2}} \partial_{x} H^{a}(x, t) d x\right| \\
& \leq \int_{x_{1}}^{x_{2}}\left|\partial_{x} H^{a}(x, t)\right| d x \leq k_{2} .
\end{aligned}
$$

If we are working on $\Sigma=R^{1}$, we can choose $x_{1}$ outside the support of $H^{a}(x, t)$ for all $t \in I$, and therefore it follows from (115) that $\left|H^{a}(x, t)\right| \leq k_{2}$ for all $(x, t) \in \Sigma \times I$. Hence, $H^{a}(x, t)$ is bounded on $\Sigma \times I$.

If instead we are working on $\Sigma=S^{1}$, we need to do more to bound $H^{a}(x, t)$. Consider $\int_{S^{1}} H^{a}(x, t) d x$, which is the spatial average of $H^{a}$ on $S^{1}$. 
From the fundamental theorem of calculus, and from the evolution equation (89), we obtain (for $t \in I$ )

$$
\begin{aligned}
\int_{S^{1}} H^{a}(x, t) d x & =\int_{t_{0}}^{t} \frac{d}{d s} \int_{S^{1}} H^{a}(x, s) d x d s+\int_{S^{1}} H^{a}\left(x, t_{0}\right) d x \\
& =-\int_{t_{0}}^{t} \int_{S^{1}} C_{b c}{ }^{a} B^{b}(x, s) H^{c}(x, s) d x d s+\int_{S^{1}} H^{a}\left(x, t_{0}\right) d x
\end{aligned}
$$

Next, using standard quadratic algebraic inequalities, we note that $\int_{S^{1}} C_{b c}{ }^{a} B^{b} H^{c} d x$ is bounded in terms of the energy,

$$
\left|\int_{S^{1}} C_{b c}^{a} B^{b} H^{c} d x\right| \leq k_{3} \mathcal{E}(t)=k_{3} \mathcal{E}\left(t_{0}\right)
$$

for some constant $k_{3}$. Hence, $\int_{t_{0}}^{t} \int_{S^{1}} C_{b c}{ }^{a} B^{b}(x, s) H^{c}(x, s) d x d s$ is bounded above and below,

$$
\left|\int_{t_{0}}^{t} \int_{S^{1}} C_{b c}{ }^{a} B^{b}(x, s) H^{c}(x, s) d x d s\right| \leq\left(t-t_{0}\right) k_{3} \mathcal{E}\left(t_{0}\right) \leq k_{4}
$$

for some constant $k_{4}$, for all $t \in I$. Then since $\int_{S^{1}} H^{a}\left(x, t_{0}\right) d x$ involves initial data only, it also is bounded above and below. Therefore, from (116) we have that

$$
\left|\int_{S^{1}} H^{a}(x, t) d x\right| \leq k_{5}
$$

and so the average of $H^{a}$ over $S^{1}$ is bounded above and below, for all $t \in I$. Combining this result with the spatial variance control (115), we conclude that $H^{a}(x, t)$ is bounded (above and below) on $\Sigma \times I$.

\section{Step 3: Bounded $E^{a}$ and $B^{a}$}

While standard $1+1$ light cone arguments do not directly apply to the system (87) to (90), a modified argument can be used with the pointwise bounds on $H^{a}$ achieved in Step 2.

Using the null form of the stress-energy conservation laws (106)-(107), along with the expressions (102)-(105) for the stress-energy components, we have

$$
\begin{aligned}
\partial_{n}(B+E)^{2}= & 2 \partial_{\ell} H^{2}-\lambda\left(v_{t}+v_{x}\right) p_{a b} \partial_{n}\left(H^{a}\left(B^{b}+E^{b}\right)\right) \\
& +\lambda\left(v_{t}+v_{x}\right) p_{a b} \partial_{\ell}\left(H^{a}\left(-B^{b}+E^{b}\right)\right)
\end{aligned}
$$




$$
\begin{aligned}
\partial_{\ell}(-B+E)^{2}= & 2 \partial_{n} H^{2}-\lambda\left(-v_{t}+v_{x}\right) p_{a b} \partial_{n}\left(H^{a}\left(B^{b}+E^{b}\right)\right) \\
& +\lambda\left(-v_{t}+v_{x}\right) p_{a b} \partial_{\ell}\left(H^{a}\left(-B^{b}+E^{b}\right)\right) .
\end{aligned}
$$

We use the field equations (87) to (90) to remove all of the derivatives which appear on the right-side of these equations. Thus

$$
\begin{aligned}
\partial_{n}(B+E)^{2}= & -2 H^{b} H^{c}\left(B^{a}+E^{a}\right) C_{a b c} \\
& -2 \lambda\left(v_{t}+v_{x}\right) H^{a}\left(-B^{b}+E^{b}\right)\left(B^{c}+E^{c}\right) Q_{b c a}
\end{aligned}
$$

and

$$
\begin{aligned}
\partial_{\ell}(-B+E)^{2}= & -2 H^{b} H^{c}\left(-B^{a}+E^{a}\right) C_{a b c} \\
& -2 \lambda\left(-v_{t}+v_{x}\right) H^{a}\left(-B^{b}+E^{b}\right)\left(B^{c}+E^{c}\right) Q_{b c a} .
\end{aligned}
$$

It is convenient here to let $\alpha^{a}:=B^{a}+E^{a}$ and $\beta^{a}:=-B^{a}+E^{a}$, and so we have

$$
\partial_{n} \alpha^{2}=-2 C_{a b c} H^{b} H^{c} \alpha^{a}-2 \lambda\left(v_{t}+v_{x}\right) Q_{b c a} H^{a} \beta^{b} \alpha^{c}
$$

and

$$
\partial_{\ell} \beta^{2}=-2 C_{a b c} H^{b} H^{c} \beta^{a}-2 \lambda\left(-v_{t}+v_{x}\right) Q_{b c a} H^{a} \beta^{b} \alpha^{c} .
$$

Since $C_{a b c}, Q_{b c a}, \lambda, v_{t}$ and $v_{x}$ are constant, and since $H^{a}$ is bounded on $\Sigma \times I$, we immediately have the following estimates for the right-sides of (124) and (125):

$$
\partial_{n} \alpha^{2} \leq k_{6} \sqrt{\alpha^{2}}+k_{7} \sqrt{\alpha^{2}} \sqrt{\beta^{2}}
$$

and

$$
\partial_{\ell} \beta^{2} \leq k_{8} \sqrt{\beta^{2}}+k_{9} \sqrt{\alpha^{2}} \sqrt{\beta^{2}}
$$

with some constants $k_{6}, k_{7}, k_{8}$, and $k_{9}$.

We now apply a light cone argument to the differential inequalities (126) and (127). First, choose an arbitrary point $(\hat{x}, \hat{t})$ in $\Sigma \times I$ to the future of the initial surface $\Sigma$, so $\hat{t}>t_{0}$, and integrate $\alpha^{2}$ back along the light ray parallel 
to $\partial_{n}$ via (126) and also integrate $\beta^{2}$ back along the light ray parallel to $\partial_{\ell}$ via (127). This yields

$$
\begin{aligned}
\alpha^{2}(\hat{x}, \hat{t}) \leq & \alpha^{2}\left(\hat{x}+\hat{t}-t_{0}, t_{0}\right)+k_{6} \int_{t_{0}}^{\hat{t}} \sqrt{\alpha^{2}(\hat{x}+\hat{t}-s, s)} d s \\
& +k_{7} \int_{t_{0}}^{\hat{t}} \sqrt{\alpha^{2}(\hat{x}+\hat{t}-s, s)} \sqrt{\beta^{2}(\hat{x}+\hat{t}-s, s)} d s
\end{aligned}
$$

and

$$
\begin{aligned}
\beta^{2}(\hat{x}, \hat{t}) \leq & \beta^{2}\left(\hat{x}+t_{0}-\hat{t}, t_{0}\right)+k_{8} \int_{t_{0}}^{\hat{t}} \sqrt{\beta^{2}(\hat{x}-\hat{t}+s, s)} d s \\
& +k_{9} \int_{t_{0}}^{\hat{t}} \sqrt{\alpha^{2}(\hat{x}-\hat{t}+s, s)} \sqrt{\beta^{2}(\hat{x}-\hat{t}+s, s)} d s .
\end{aligned}
$$

Next, take the supremum of these expressions over $\Sigma$. Letting $\hat{\alpha}^{2}(t):=$ $\sup _{x \in \Sigma} \alpha^{2}(x, t)$ and $\hat{\beta}^{2}(t):=\sup _{x \in \Sigma} \beta^{2}(x, t)$, we obtain from (128)

$$
\begin{aligned}
\hat{\alpha}^{2}(\hat{t}) \leq & \hat{\alpha}^{2}\left(t_{0}\right)+k_{6} \sup _{x \in \Sigma} \int_{t_{0}}^{\hat{t}} \sqrt{\alpha^{2}(x, s)} d s \\
& +k_{7} \sup _{x \in \Sigma} \int_{t_{0}}^{\hat{t}} \sqrt{\alpha^{2}(x, s)} \sqrt{\beta^{2}(x, s)} d s \\
\leq & \hat{\alpha}^{2}\left(t_{0}\right)+k_{6} \int_{t_{0}}^{\hat{t}} \sqrt{\hat{\alpha}^{2}(s)} d s+k_{7} \int_{t_{0}}^{\hat{t}} \sqrt{\hat{\alpha}^{2}(s)} \sqrt{\hat{\beta}^{2}(s)} d s \\
\leq & \hat{\alpha}^{2}\left(t_{0}\right)+k_{10}\left(\hat{t}-t_{0}\right)^{1 / 2}\left(\int_{t_{0}}^{\hat{t}} \hat{\alpha}^{2}(s) d s\right)^{1 / 2} \\
& +k_{11}\left(\int_{t_{0}}^{\hat{t}} \hat{\alpha}^{2}(s) d s\right)^{1 / 2}\left(\int_{t_{0}}^{\hat{t}} \hat{\beta}^{2}(s) d s\right)^{1 / 2}
\end{aligned}
$$

where the last step is a consequence of the Holder inequality. If we define

$$
a(t):=\int_{t_{0}}^{t} \hat{\alpha}^{2}(s) d s
$$

and

$$
b(t):=\int_{t_{0}}^{t} \hat{\beta}^{2}(s) d s
$$


then $(130)$ can be written as (with $\hat{t}$ replaced by $t$ )

$$
\frac{d}{d t} a(t) \leq a\left(t_{0}\right)+k_{10}\left(t-t_{0}\right)^{1 / 2} a^{1 / 2}(t)+k_{11} a^{1 / 2}(t) b^{1 / 2}(t) .
$$

Similarly, from (129), we derive

$$
\frac{d}{d t} b(t) \leq b\left(t_{0}\right)+k_{12}\left(t-t_{0}\right)^{1 / 2} b^{1 / 2}(t)+k_{13} a^{1 / 2}(t) b^{1 / 2}(t) .
$$

We want to show $a(t)$ and $b(t)$ are bounded functions of $t$ by applying a Gronwall type argument to the coupled inequalities (133),(134). It is useful first to divide by $a^{1 / 2}(t)$ in (133) and by $b^{1 / 2}(t)$ in (134), yielding

$$
\begin{aligned}
\frac{d}{d t} a^{1 / 2}(t) & \leq a\left(t_{0}\right) a^{-1 / 2}(t)+k_{10}\left(t-t_{0}\right)^{1 / 2}+k_{11} b^{1 / 2}(t), \\
\frac{d}{d t} b^{1 / 2}(t) & \leq b\left(t_{0}\right) b^{-1 / 2}(t)+k_{12}\left(t-t_{0}\right)^{1 / 2}+k_{13} a^{1 / 2}(t) .
\end{aligned}
$$

We estimate the term $a\left(t_{0}\right) a^{-1 / 2}(t)$ by using the fact that $a(t)$ is a monotonic increasing function of $t$, due to positivity of $\hat{\alpha}^{2}$ in (131). Thus, $a\left(t_{0}\right) a^{-1 / 2}(t)$ is bounded by $a^{-1 / 2}\left(t_{0}\right)$. In addition, we note the term $k_{10}\left(t-t_{0}\right)^{1 / 2}$ is bounded since $t \in I$ is bounded. We thereby obtain

$$
\frac{d}{d t} a^{1 / 2}(t) \leq k_{14}+k_{11} b^{1 / 2}(t)
$$

Similarly, we obtain

$$
\frac{d}{d t} b^{1 / 2}(t) \leq k_{15}+k_{13} a^{1 / 2}(t)
$$

Adding (137) and (138), and defining $c(t):=a^{1 / 2}(t)+b^{1 / 2}(t)$, we derive

$$
\frac{d}{d t} c(t) \leq k_{18}+k_{17} c(t)
$$

Gronwall's inequality immediately applies to (139), and so we determine that $c(t)$ is bounded for all $t \in I$. Then $a^{1 / 2}(t)$ and $b^{1 / 2}(t)$, which are positive, are bounded.

Returning to the inequalities (133)-(134), it follows that $\frac{d}{d t} a(t)$ and $\frac{d}{d t} b(t)$ are each bounded. Hence, from the definitions of $a$ and $b$, we obtain that $\sup _{\Sigma} \alpha^{2}$ and $\sup _{\Sigma} \beta^{2}$ are bounded for all $t \in I$. Since $\alpha^{2}=\left(B^{a}+E^{a}\right)^{2}$ and $\beta^{2}=\left(-B^{a}+E^{a}\right)^{2}$, we conclude that $B^{a}(x, t)$ and $E^{a}(x, t)$ are bounded on $\Sigma \times I$. 


\section{Step 4: Bounded Derivatives}

Now that we have determined that $E^{a}, H^{a}$, and $B^{a}$ are bounded on $\Sigma \times I$, we proceed to show that the first derivatives of these functions, and subsequently all higher order derivatives, are bounded on $\Sigma \times I$.

We start with $H^{a}$. From (87) and (89), it follows that since $E^{a}, H^{a}$, and $B^{a}$ are bounded, then $\partial_{x} H^{a}$ and $\partial_{t} H^{a}$ are bounded. Similarly, if the order $n$ derivatives of $E^{a}, H^{a}$, and $B^{a}$ are bounded, then it follows from (derivatives of ) (87) and (89) that the order $n+1$ derivatives of $H^{a}$ are bounded. Hence, (by induction on $n$ ), the derivatives of $H^{a}$ to all orders are bounded.

For $E^{a}$ and $B^{a}$, we use light cone arguments much like step 3 , but involving a "derivative stress-energy" tensor. Specifically, let

$$
\begin{gathered}
T_{(1) \ell \ell}=\frac{1}{4}\left(\partial_{x} B+\partial_{x} E\right)^{2}+\frac{\lambda}{4}\left(v_{t}+v_{n}\right) \partial_{x} H^{a}\left(\partial_{x} B^{b}+\partial_{x} E^{b}\right) p_{a b} \\
T_{(1) n n}=\frac{1}{4}\left(-\partial_{x} B+\partial_{x} E\right)^{2}-\frac{\lambda}{4}\left(-v_{t}+v_{n}\right) \partial_{x} H^{a}\left(-\partial_{x} B^{b}+\partial_{x} E^{b}\right) p_{a b} \\
T_{(1) \ell n}=-\frac{1}{2}\left(\partial_{x} H\right)^{2}+\frac{\lambda}{4}\left(-v_{t}+v_{n}\right) \partial_{x} H^{a}\left(\partial_{x} B^{b}+\partial_{x} E^{b}\right) p_{a b} \\
T_{(1) n \ell}=-\frac{1}{2}\left(\partial_{x} H\right)^{2}-\frac{\lambda}{4}\left(v_{t}+v_{n}\right) \partial_{x} H^{a}\left(-\partial_{x} B^{b}+\partial_{x} E^{b}\right) p_{a b}
\end{gathered}
$$

as defined analogously to the stress-energy components (102) to (105). Then, as a consequence of the field equations (87) to (90), we find

$$
\partial_{n} T_{(1) \ell \ell}+\partial_{\ell} T_{(1) n \ell}=Y_{1}\left(E, H, B ; \partial_{x} E, \partial_{x} B\right)
$$

and

$$
\partial_{\ell} T_{(1) n n}+\partial_{n} T_{(1) \ell n}=Y_{2}\left(E, H, B ; \partial_{x} E, \partial_{x} B\right)
$$

where $Y_{1}$ and $Y_{2}$ are homogeneous quadratic in $\partial_{x} E$ and $\partial_{x} B$, with bounded coefficients. Although (144) and (145) are not strict conservation equations, we can nevertheless proceed similarly to step 3 . 
Through use of the field equations, we can express (144) and (145) as

$$
\partial_{n}\left(\partial_{x} B+\partial_{x} E\right)^{2}=\tilde{Y}_{1}\left(E, H, B ; \partial_{x} E, \partial_{x} B\right)
$$

and

$$
\partial_{\ell}\left(-\partial_{x} B+\partial_{x} E\right)^{2}=\tilde{Y}_{2}\left(E, H, B ; \partial_{x} E, \partial_{x} B\right)
$$

with $\tilde{Y}_{1}$ and $\tilde{Y}_{2}$ of the same nature as $Y_{1}$ and $Y_{2}$. It then follows from standard algebraic inequalities that

$$
\begin{gathered}
\partial_{n}\left(\partial_{x} B+\partial_{x} E\right)^{2} \leq k_{19}\left|\partial_{x} B\right|^{2}+k_{20}\left|\partial_{x} E\right|^{2} \\
\partial_{\ell}\left(-\partial_{x} B+\partial_{x} E\right)^{2} \leq k_{21}\left|\partial_{x} B\right|^{2}+k_{22}\left|\partial_{x} E\right|^{2} .
\end{gathered}
$$

We now apply the light cone arguments of step 3 to the differential inequalities (148) and (149): Starting at an arbitrary point in $\Sigma \times I$, we inte-

grate (148) and (149) back to the initial surface along light rays generated by $\partial_{n}$ and $\partial_{\ell}$. Taking suprema over $\Sigma$ and adding the resulting inequalities, we obtain

$$
\begin{aligned}
\sup _{x \in \Sigma}[ & \left.\left(\partial_{x} B(x, t)\right)^{2}+\left(\partial_{x} E(x, t)\right)^{2}\right] \leq \\
& k_{23}+k_{24} \int_{t_{0}}^{t} \sup _{x \in \Sigma}\left[\left(\partial_{x} B(x, s)\right)^{2}+\left(\partial_{x} E(x, s)\right)^{2}\right] d s .
\end{aligned}
$$

Applying the Gronwall inequality to (150) shows that $\left|\partial_{x} B\right|$ and $\left|\partial_{x} E\right|$ are bounded for all $t \in I$. With $E^{a}, H^{a}, B^{a}, \partial_{x} E^{a}$ and $\partial_{x} B^{a}$ bounded, it follows from the evolution equations (88) and (90) that $\partial_{t} E^{a}$ and $\partial_{t} B^{a}$ are bounded as well.

The previous argument can be applied to all orders of derivatives of the fields. This establishes that $E^{a}, H^{a}, B^{a}$, and all of their derivatives are bounded on $\Sigma \times I$. Global existence now follows from the usual "open-closed" arguments.

\section{GLOBAL EXISTENCE FOR EQUIVARIANT FRAME FIELD EQUATIONS WITH TORSION}

As discussed in Section 3, while left-equivariant wave maps (12) correspond to invariant frame fields (61), conjugate-equivariant wave maps (14) and rightequivariant wave maps (13) correspond to equivariant frame fields (62). In 
this section we show that global existence holds for smooth solutions to the Cauchy problem for translation equivariant frame fields.

We first note that by definition of translation equivariance, $K_{\mu}^{a}(x, y, t)$ can be expressed as

$$
\begin{aligned}
& K_{x}^{a}(x, y, t)=\exp (-y R) E^{a}(x, t) \exp (y R) \\
& K_{y}^{a}(x, y, t)=\exp (-y R) H^{a}(x, t) \exp (y R) \\
& K_{t}^{a}(x, y, t)=\exp (-y R) B^{a}(x, t) \exp (y R)
\end{aligned}
$$

in terms of some Lie-algebra valued fields $\left\{E^{a}(x, t), H^{a}(x, t), B^{a}(x, t)\right\}$ which do not depend on $y$. Here $R$ is a fixed (constant) element in the Lie algebra; the left multiplication by $\exp (-y R)$ combined with right multiplication by $\exp (y R)$ denotes the adjoint action of a one-parameter Lie subgroup on the Lie algebra.

Substituting expressions (151) to (153) into the frame field equations with torsion (56) and (58) on Minkowski space, we obtain the following $1+1$ reduced PDE system

$$
\begin{aligned}
\partial_{x} H^{a}= & -C_{b c}{ }^{a} E^{b}\left(H^{c}-R^{c}\right) \\
\partial_{t} E^{a}= & \partial_{x} B^{a}-C_{b c}{ }^{a} B^{b} E^{c} \\
\partial_{t} H^{a}= & -C_{b c}{ }^{a} B^{b}\left(H^{c}-R^{c}\right) \\
\partial_{t} B^{a}= & \partial_{x} E^{a}-C_{b c}{ }^{a} H^{b} R^{c}-C^{a}{ }_{b c}\left(B^{b} B^{c}-E^{b} E^{c}-H^{b} H^{c}\right) \\
& \quad-\lambda Q^{a}{ }_{b c}\left(v_{y} B^{b} E^{c}-v_{x} B^{b} H^{c}+v_{t} E^{b} H^{c}\right)
\end{aligned}
$$

provided that $C^{a}{ }_{b c}$ and $Q^{a}{ }_{b c}$ are invariant under the adjoint action of $\exp (y R)$. We note that the only difference between these equations for translation equivariant frame fields and equations (87) to (90) for translation invariant frame fields is the presence of the commutator terms involving $R$.

While the expressions for the field equations are changed somewhat in passing from invariant to equivariant frame fields, the expressions for the stress-energy components (93) to (100) and (102) to (105) do not change at all. (In particular, while $K_{\mu}^{a}$ is not independent of $y$, the quadratic expressions $K_{\mu}^{a} K_{\nu}^{b} g_{a b}$ and $K_{\mu}^{a} K_{\nu}^{b} p_{a b}$ are invariant, and consequently so is $T_{\mu \nu}$. )

Initial data for the Cauchy problem for translation equivariant frame fields consists of Lie-algebra valued functions $\left\{\hat{E}^{a}(x), \hat{H}^{a}(x), \hat{B}^{a}(x)\right\}$ on $\Sigma$ which satisfy the constraint

$$
\partial_{x} \hat{H}^{a}=-C_{b c}{ }^{a} \hat{E}^{b}\left(\hat{H}^{c}-R^{c}\right) .
$$


A solution to the Cauchy problem is a set of fields $\left\{E^{a}(x, t), H^{a}(x, t), B^{a}(x, t)\right\}$ satisfying equations (154) to (157) and the initial conditions (92).

The global existence result, and its corollary, are stated as follows. Let $\Sigma$ denote $R^{1}$ or $S^{1}$, and introduce coordinates $(x, t)$ for $\Sigma \times R^{1}$. Fix constants $v_{t}, v_{x}, v_{y}$. Let $G$ be a Lie group and let $R^{a}$ be a fixed (constant) vector in the Lie algebra of $G$. Assume $G$ admits on its Lie algebra a positive definite metric tensor $g_{a b}$ and a skew tensor $p_{a b}$ which are each invariant under the adjoint action of the Lie subgroup generated by $R^{a}$ :

$$
\begin{aligned}
& g_{a e} C_{b c}{ }^{e} R^{c}=-g_{b e} C_{a c}^{e} R^{c} \\
& p_{a e} C_{b c}{ }^{e} R^{c}=p_{b e} C_{a c}{ }^{e} R^{c}
\end{aligned}
$$

where $C_{b c}{ }^{a}$ denotes the Lie-algebra commutator structure tensor. Let $Q^{a}{ }_{b c}$ be the tensor defined by (54).

Theorem 4. Let $\lambda$ be a small constant. ${ }^{4}$ For any smooth compact support initial data (98) satisfying (158), the Cauchy problem (154) to (157) has a unique smooth global solution $\left\{E^{a}(x, t), H^{a}(x, t), B^{a}(x, t)\right\}$ for all $t \in R^{1}$.

From Propositions 2 and 3 we obtain a corresponding result for wave maps.

Theorem 5. The Cauchy problems for conjugate-translation equivariant wave maps (14) and for right-translation equivariant wave maps (13), with or without torsion, have unique smooth global solutions for all smooth compact support initial data.

Remark 2: Under the translation invariant form (61) for frame fields, which corresponds to left-translation equivariant (12) or translation invariant (10) wave maps, the reduction of the frame field equations and corresponding wave map equation is consistent for any Lie group target with $(G, g, p)$ invariant under left multiplication. However, this is not the case under the translation equivariant form (62) for frame fields, which corresponds to conjugatetranslation equivariant (14) or right-translation equivariant (12) wave maps. The translation equivariance ansatz gives a consistent reduction of the frame field equations and corresponding wave map equation only if the target geometry $(G, g, p)$ is invariant under right multiplication by the translation group 
generated by the Lie algebra element $R$ appearing in (12) to (14) for wave maps and (62) for frame fields. We refer to this condition, given by (159) and (160), as translation invariance of the target. As shown in Proposition A in the appendix, every compact semi-simple Lie group $G$ admits a translation invariant geometry $(G, g, p)$, except that the dimension of $G$ must be greater than three to support a non-zero torsion $Q$ (see Remark 1).

\section{Proof of Theorem 4:}

The proof of Theorem 4 is very similar to that of Theorem 2. We summarize the differences (if any) in each step.

\section{Step 1: Conserved Energy}

Since the expression for the energy is unchanged and since it is conserved, there are no changes in obtaining $L^{2}$ bounds for $E^{a}(x, t), H^{a}(x, t), B^{a}(x, t)$.

\section{Step 2: Bounded $H^{a}$}

Instead of (112), we have

$$
\int_{\Sigma}\left|\partial_{x} H^{a}\right| d x \leq \int_{\Sigma}\left|C_{b c}{ }^{a} E^{b} H^{c}\right| d x+\int_{\Sigma}\left|C_{b c}{ }^{a} E^{b} R^{c}\right| d x .
$$

The first of the two terms on the right hand side of (161) may be handled as in $(\sqrt{113})$. As for the second term, we have

$$
\begin{aligned}
\int_{\Sigma}\left|C_{b c}{ }^{a} E^{b} R^{c}\right| d x & \leq k_{25} \int_{\Sigma}\left|E^{b}\right| d x \\
& <k_{25}\left(\int_{\Sigma} E^{2} d x\right)^{1 / 2} \\
& \leq k_{26}
\end{aligned}
$$

where the second inequality uses the compact support of $E^{b}$ together with the Holder inequality, and the last inequality follows from the $L^{2}$ bound on $E^{a}$. Hence we obtain

$$
\int_{\Sigma}\left|\partial_{x} H^{a}\right| d x \leq k_{27}
$$

analogous to (114). 
If $\Sigma=R^{1}$, the argument leading to a pointwise bound on $H^{a}(x, t)$ for $t \in I$ proceeds exactly as in the proof of Theorem 2 . If $\Sigma=S^{1}$, then we need to modify the argument which begins with (116). We have

$$
\begin{aligned}
\int_{S^{1}} H^{a}(x, t) d x= & -\int_{t_{0}}^{t} \int_{S^{1}} C_{b c}^{a} B^{b}(x, s)\left(H^{c}(x, s)+R^{c}\right) d x d s \\
& +\int_{S^{1}} H^{a}\left(x, t_{0}\right) d x .
\end{aligned}
$$

The term $\int_{t_{0}}^{t} \int_{S^{1}} C^{a}{ }_{b c} B^{b}(x, s) R^{c} d x d s$ can be bounded from above using the same quadratic inequality that is used in (162), and so we obtain

$$
\left|\int_{S^{1}} H^{a}(x, t) d x\right| \leq k_{28}
$$

analogous to (119). The argument for pointwise bounds on $H^{a}(x, t)$ for $\Sigma=S^{1}$ can then be completed as in the proof of Theorem 2 .

\section{Step 3: Bounded $E^{a}$ and $B^{a}$}

From inequalities (126) and (127) onward, the light-cone arguments used to bound $E^{a}(x, t)$ and $B^{a}(x, t)$ in the proof of Theorem 2 work identically to bound $E^{a}(x, t)$ and $B^{a}(x, t)$ here. To arrive at (126) and (127) we use the following equations, analogous to (122) and (123),

$$
\begin{aligned}
\partial_{n}(B+E)^{2}= & -2 H^{b}\left(H^{c}+R^{c}\right)\left(B^{a}+E^{a}\right) C_{a b c} \\
& -2 \lambda\left(v_{t}+v_{x}\right) H^{a}\left(-B^{b}+E^{b}\right)\left(B^{c}+E^{c}\right) Q_{b c a} \\
& +2 \lambda\left(v_{t}-v_{x}\right) C_{b c}{ }^{a} R^{b}\left(B^{c}+E^{c}\right)\left(B^{b}-E^{b}\right) p_{a b}, \\
\partial_{n}(-B+E)^{2}= & -2 H^{b}\left(H^{c}+R^{c}\right)\left(-B^{a}+E^{a}\right) C_{a b c} \\
& -2 \lambda\left(-v_{t}+v_{x}\right) H^{a}\left(-B^{b}+E^{b}\right)\left(B^{c}+E^{c}\right) Q_{b c a} \\
& +2 \lambda\left(v_{t}+v_{x}\right) C_{b c}{ }^{a} R^{b}\left(-B^{c}+E^{c}\right)\left(B^{b}+E^{b}\right) p_{a b} .
\end{aligned}
$$

Adopting the notation $\alpha^{a}:=B^{a}+E^{a}, \beta^{a}:=-B^{a}+E^{a}$, these equations become

$$
\begin{aligned}
\partial_{n} \alpha^{2}= & -2 C_{a b c} H^{b}\left(H^{c}+R^{c}\right) \alpha^{a} \\
& -2 \lambda\left(v_{t}+v_{x}\right) Q_{b c a} H^{a} \beta^{b} \alpha^{c}+2 \lambda\left(v_{t}+v_{x}\right) R^{b} \alpha^{c} \beta^{b} C_{b c}{ }^{a} p_{a b},
\end{aligned}
$$




$$
\begin{aligned}
\partial_{\ell} \beta^{2}= & -2 C_{a b c} H^{b}\left(H^{c}+R^{c}\right) \beta^{a} \\
& -2 \lambda\left(-v_{t}+v_{x}\right) Q_{b c a} H^{a} \beta^{a} \alpha^{c}+2 \lambda\left(v_{t}+v_{x}\right) R^{b} \beta^{c} \alpha^{b} C_{b c}{ }^{a} p_{a b} .
\end{aligned}
$$

Then, noting that $C_{a b c}, Q_{a b c}, \lambda, v_{t}, v_{x}$ and $R$ are constant, and recalling that $H^{a}$ is bounded on $\Sigma \times I$, we obtain

$$
\partial_{n} \alpha^{2} \leq k_{29} \sqrt{\alpha^{2}}+k_{30} \sqrt{\alpha^{2}} \sqrt{\beta^{2}}
$$

and

$$
\partial_{\ell} \beta^{2} \leq k_{31} \sqrt{\beta^{2}}+k_{32} \sqrt{\alpha^{2}} \sqrt{\beta^{2}}
$$

which are identical to (126) and (127).

\section{Step 4: Bounded Derivatives}

One can see in Step 2 and Step 3 that the presence of the commutator terms involving $R$ in the field equations (154) to (157) changes little in the arguments for boundedness, since these extra terms are easily controlled by the analogous quadratic terms appearing in the equations. The same holds true for Step 4. We can define the derivative stress-energy components just as in (140) to (143) and then obtain conservation equations similar to (144) to (145), with small modifications in the expressions $Y_{1}$ and $Y_{2}$ which appear there. These modifications are readily handled in deriving the estimate (148) and (149). The rest of the argument proceeds unchanged.

Hence we obtain global existence.

\section{CONCLUDING REMARKS}

The wave map global existence results we have obtained here extend previous work in two significant ways. First, our study of translation equivariant critical wave maps for Lie group targets (Theorems 3 and 5) provides a counterpart to work on rotationally equivariant critical wave maps for symmetricspace targets (see [4, 6]). Second, our inclusion of torsion gives an interesting generalization of critical wave maps for arbitrary targets, which ties into cur-

rent work on integrable chiral models in $2+1$ dimensions in the case of Lie group targets [8]. 
Furthermore, our results demonstrate the utility of the frame formulation of wave maps for Lie group targets (Proposition 1). The translationequivariant reduction of critical wave maps studied here is motivated by this formulation and the analysis is especially straightforward in terms of frames. An important question to investigate for future work is how the frame formulation might help in understanding the unreduced critical wave map equation for general Lie group targets and symmetric-space targets.

\section{APPENDIX}

Proposition A. Let $G$ be a semi-simple Lie group with commutator structure tensor $C_{b c}{ }^{a}$.

1. The Lie algebra of $G$ admits a translation invariant (159) positivedefinite metric $g_{a b}$ if $G$ is compact.

2. The Lie algebra of $G$ admits a translation invariant (160) skew-tensor $p_{a b}$ with non-zero torsion (54) if $G$ is compact and has dimension greater than three.

3. If $G$ has dimension three then the torsion (54) is zero for every skewtensor $p_{a b}$ on the Lie algebra of $G$.

Proof of 1: If $G$ is compact then its Lie algebra admits an invariant positivedefinite metric $g_{a b}$ (see, e.g. [13]), which satisfies

$$
g_{a e} C_{b c}{ }^{e}=-g_{b e} C_{a c}{ }^{e} .
$$

(In particular, the Cartan-Killing metric given by $g_{a b}:=-C_{a e}{ }^{c} C_{b c}{ }^{e}$ is both invariant and positive-definite.) Hence condition (159) holds.

Proof of 2 and 3: Hereafter $g_{a b}$ denotes the Cartan-Killing metric. We first remark that, for any $G$, the natural construction

$$
p_{a b}:=C_{a b}{ }^{d} g_{d e} R^{e}
$$

is easily seen to yield a translation invariant skew-tensor. But the resulting torsion tensor (54) is always zero, since

$$
p_{e[a} C_{b c]}^{e}=C_{e[a}^{d} C_{b c]}{ }^{e} g_{d f} R^{f}=0
$$


by the Jacobi identity.

In three dimensions it is easy to show that $C_{a b}{ }^{e} g_{e c}$ must be proportional to the totally-skew Levi-Civita tensor $\epsilon_{a b c}$, while any skew-tensor $p_{a b}$ can be expressed in the form

$$
p_{a b}=\epsilon_{a b c} p^{c}
$$

for some vector $p^{c}$ in the Lie algebra of $G$. Thus, it follows that $p_{a b}$ must have the form (173) where $R^{e}$ is proportional to $p^{e}$, and hence from (173) and (174) we have that the torsion tensor (54) is zero. This shows that there is no torsion for any three-dimensional $G$ (and hence none in particular with $p_{a b}$ being translation invariant).

Now suppose $G$ has dimension greater than three. In this case, $G$ must have rank greater than one and hence the Lie algebra of $G$ possesses an abelian subalgebra of dimension at least two (see, e.g. [13]). This allows the explicit construction of a translation invariant skew-tensor $p_{a b}$ as follows. Let $p^{a}, q^{a}$ be any two (linearly independent) commuting vectors in the Lie algebra of $G$, so $p^{a} q^{b} C_{a b}{ }^{c}=0$, and let $p_{e}:=g_{e a} p^{a}, q_{e}:=g_{e a} q^{a}$. Set $R^{a}:=\alpha p^{a}+\beta q^{a} \neq 0$ with constants $\alpha, \beta$. Then it is straightforward to show that the skew-tensor defined by

$$
p_{a b}:=2 p_{[a} q_{b]}
$$

is translation invariant as a consequence of $p$ and $q$ commuting with $R$. Now it remains to show that the torsion tensor given by (54) and (176) is non-zero.

We have

$$
g_{a d} Q_{b c}^{d}=3 / 2\left(p_{e} q_{[a} C_{b c]}^{e}-q_{e} p_{[a} C_{b c]}^{e}\right) .
$$

To show that the tensor (177) is non-zero when $G$ is compact, we contract (177) with the vector $s^{a}=p^{a} q^{e} q_{e}-q^{a} p^{e} q_{e}$ satisfying $s^{a} q_{a}=0$. This yields

$$
s^{a} g_{a d} Q_{b c}^{d}=-1 / 2 s^{a} p_{a} C_{b c}{ }^{e} q_{e} .
$$

with $s^{a} p_{a}=p^{a} p_{a} q^{d} q_{d}-\left(p^{a} q_{a}\right)^{2} \neq 0$ due to positive-definiteness of $g_{a b}$. Moreover, since $G$ is semi-simple, its Lie algebra has empty center and so $C_{b c}{ }^{e} q_{e}=C_{e b}{ }^{a} q^{e} g_{c a}$ is non-zero (that is, there exists a vector $v^{b}$ so that $\left.C_{e b}{ }^{a} q^{e} v^{b} \neq 0\right)$. Therefore, $s^{a} g_{a d} Q_{b c}^{d}$ is non-zero and thus so is the torsion tensor (177). 


\section{ACKNOWLEDGEMENTS}

Various portions of this work were carried out at the Courant Institute and at the University of Washington. Partial support for this research has come from NSF grant PHY-9800732 at the University of Oregon.

\section{References}

[1] Gu, Chao-hao "On the Cauchy Problem for Harmonic Maps Defined on Two-Dimensional Minkowski Space" Comm. Pure Appl. Math. $\underline{32}$ (1980), 727-737.

[2] Genibre, J. and Velo, G. "The Cauchy Problem for the $O(N), C P(N-$ $1)$, and $G C(N, p)$ Models" Ann. Phys. 142 (1982), 393-415.

[3] Shatah, J. "Weak solutions and development of singularities of SU(2) $\sigma$-model" Comm. Pure Appl. Math. $\underline{41}$ (1988), 459-469.

[4] Shatah, J. and Tahveldar-Zadeh, S. "On the Cauchy Problem for Equivariant Wave Maps" Comm. Pure Appl. Math. $\underline{67}$ (1994), 719754 .

[5] Klainerman, S. "On the Regularity of Classical Field Theories in Minkowski Space Time $R^{3+1}$ " in Nonlinear Partial Differential Equations in Geometry and Physics [eds: Baker and Freise], Birkhäuser (1997).

[6] Shatah, J. and Struwe, M. Geometric Wave Equations, Courant Lecture Notes in Mathematics \#2 (1998).

[7] Christodoulou, D. and Tahveldar-Zadeh, S. "On the Regularity of Spherically Symmetric Wave maps" Comm. Pure Appl. Math. $\underline{66}$ (1993), 1042-1091.

[8] Ward, R. S. "Soliton solutions in an integrable chiral model in $2+1$ dimensions" J. Math. Phys. 29 (1988), 386-389.

[9] Sogge, C. Lectures on Nonlinear Wave Equations, International Press, Cambridge (1995). 
[10] Anco, S. "New Spin-One Gauge Theory in Three Dimensions" J. Math. Phys. $\underline{36}$ (1995), 6553-6569.

[11] Anco, S. "Novel generalization of three-dimensional Yang-Mills theory" J. Math. Phys. $\underline{38}$ (1997), 3399-3413.

[12] Anco, S. and Isenberg, J. "Cauchy problem for generalizations of YangMills theory", in preparation (2000).

[13] Sagle, A. A. and Walde, R. E. Introduction to Lie Groups and Lie Algebras, Academic Press (1973). 